\title{
Papers that matter
}

\author{
Nature Geoscience aims to publish important science, but the journal also strives to offer a platform to voices driving \\ change within the geoscience community. We welcome submissions on community issues that encourage reader \\ engagement and inspire action.
}

T he killing of George Floyd by Minneapolis police - just one example of police brutality against Black people and of the racism that continues to dominate the lives of Black Americans - has triggered anti-racism protests around the world. The surging Black Lives Matter movement is a call to action and lasting change. Science is not immune to systemic racism. On 10 June, Nature Geoscience and the other Nature journals (see, for example, ref. ${ }^{1}$ ) joined \#ShutDownSTEM \#ShutDownAcademia \#Strike4BlackLives, a scientist-led initiative to kick-start a commitment to eradicate anti-Black racism in science with a day of reflection, education and planning. For one day, researchers, institutions and organizations pondered not just the problem itself, but also actions they would take to move their communities forward. We were proud to see contributors from our pages inspiring debate and spurring action in the geosciences, and hope to hear more voices bringing about change in the future.

The days leading up to and following 10 June saw two Comments published in Nature Geoscience being shared repeatedly in discussions among geoscientists on social media. The first is a 2018 paper by Rachel Bernard and Emily Cooperdock ${ }^{2}$ that shows little improvement in the diversity of geosciences in the United States over the past four decades, despite outreach efforts aimed at shifting the demographics. There has been some progress on gender balance, but in 2016 minority groups comprised $31 \%$ of the US population, yet received only $6 \%$ of geoscience doctorates awarded to US citizens and permanent residents - the lowest proportion of all science, technology, engineering and mathematics fields. Bernard and Cooperdock provide undeniable evidence that the geosciences have huge and persistent problems with inclusion and representation. This is the sobering foundation that geoscientists need to reckon with as we seek to educate ourselves about the uncomfortable reality of our community.

Confronting systemic racism in our community requires more than education: it demands action. For those with research skills, education is relatively easy. Social action, on the other hand, requires a

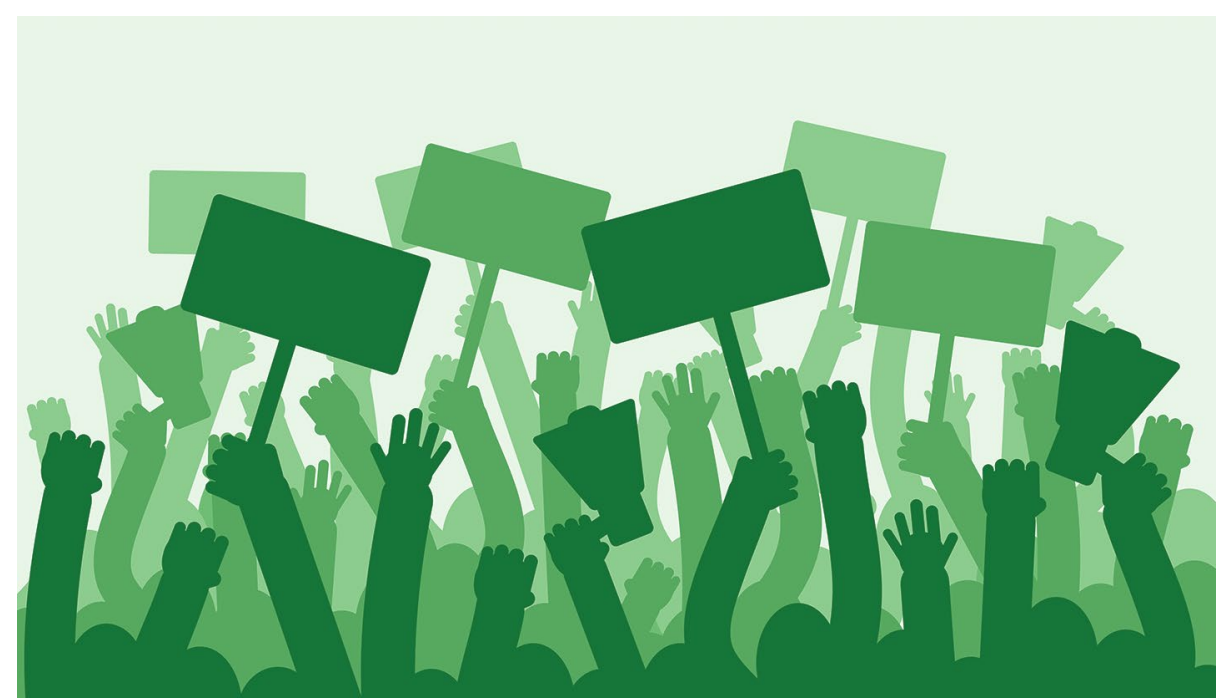

Credit: Aleksei Egorov / Alamy Stock Vector

different skill set and can be daunting. Recognizing one's own responsibility when the problem does not obviously affect you personally can be difficult, and bringing about real change requires the majority not only the burdened minority - to take up the cause. A Comment by Kuheli Dutt on racism in the geosciences ${ }^{3}$, published at the end of 2019, directly challenges white geoscientists to take personal responsibility for the diversity shortcomings of the field and undertake action to combat racism and promote inclusion.

As the Black Lives Matter protests gained momentum, the page views of the Dutt paper skyrocketed. Papers are usually hottest when they first come out, not half a year later, but this paper had clearly struck a chord. It was remarkable that geoscientists were not just reading the piece and finding it interesting, but sharing the piece, discussing it with colleagues, and using it to design plans for change. The paper provides a road map to action, and geoscientists were following its lead.

This sort of engagement is difficult to measure with standard metrics of impact for a publication such as citations, clicks, or news coverage, but there is no doubting the impact that Dutt's paper has had on the geoscience community.
We want to continue to publish papers on important community issues that actively engage geoscientists and inspire constructive discussion and action. We welcome such submissions. We also want to represent many different voices and experiences on topics such as diversity and inclusion, which for us has been rather United States- and Western Europe-centric so far.

Nature Geoscience aims to publish papers that will be important to geoscientists, whether that is because they are important science or important for the community. Giving a platform to the latter will promote the former: advances in geoscience rely on a diverse and inclusive community ${ }^{4}$. When it comes to papers on community issues whether opinion pieces or original research - we are looking for high impact. By that, we do not mean high numbers of citations, but a high level of engagement with, and action from, geoscientists.

Published online: 2 July 2020

https://doi.org/10.1038/s41561-020-0613-2

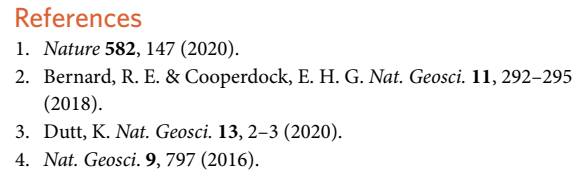

2. Bernard, R. E. \& Cooperdock, E. H. G. Nat. Geosci. 11, 292-295 (2018).

3. Dutt, K. Nat. Geosci. 13, 2-3 (2020).

4. Nat. Geosci. 9, 797 (2016). 